\title{
A Balanced Ensemble Approach to Weighting Classifiers for Text Classification
}

\author{
Gabriel Pui Cheong Fung ${ }^{1}$, Jeffrey Xu Yu ${ }^{1}$, Haixun Wang ${ }^{2}$, David W. Cheung ${ }^{3}$, Huan Liu ${ }^{4}$ \\ 1 The Chinese University of Hong Kong, Hong Kong, China, \{pcfung, yu\}@ se.cuhk.edu.hk \\ 2 IBM T. J. Watson Research Center, New York, USA, haixun@us.ibm.com \\ 3 The University of Hong Kong, Hong Kong, China, dcheung@cs.hku.hk \\ 4 Arizona State University, Arizona, USA, hliu@asu.edu
}

\begin{abstract}
This paper studies the problem of constructing an effective heterogeneous ensemble classifier for text classification. One major challenge of this problem is to formulate a good combination function, which combines the decisions of the individual classifiers in the ensemble. We show that the classification performance is affected by three weight components and they should be included in deriving an effective combination function. They are: (1) Global effectiveness, which measures the effectiveness of a member classifier in classifying a set of unseen documents; (2) Local effectiveness, which measures the effectiveness of a member classifier in classifying the particular domain of an unseen document; and (3) Decision confidence, which describes how confident a classifier is when making a decision when classifying a specific unseen document. We propose a new balanced combination function, called Dynamic Classifier Weighting (DCW), that incorporates the aforementioned three components. The empirical study demonstrates that the new combination function is highly effective for text classification.
\end{abstract}

\section{Introduction}

Let $\mathcal{U}$ be a set of unseen documents and $\mathcal{C}$ be a set of predefined categories. Automated text classification is the process of labeling $\mathcal{U}$ with $\mathcal{C}$, such that every $d \in \mathcal{U}$ will be assigned to some of the categories in $C$. Note that $d$ can be assigned to none of the categories in $\mathcal{C}$. If the number of categories in $C$ is more than two $(|C|>2)$, it is a multilabel text classification problem. Since every multi-label text classification problem can be transformed to a binarylabel text classification problem, we focus on the binary problem in this paper $(|\mathcal{C}|=2)$. Let $c \in \mathcal{C}$. Binary-label text classification is to construct a binary classifier, denoted by $\Phi(\cdot)$, for $c$ such that:

$$
\Phi(d)= \begin{cases}1 & \text { if } f(d)>0 \\ -1 & \text { otherwise }\end{cases}
$$

where $\Phi(d)=1$ indicates that $d$ belongs to $c$ and $\Phi(d)=$ -1 indicates that $d$ does not belong to it. $f(\cdot) \in \mathfrak{R}$ is a decision function. Every classifier, $\Phi_{i}$, has its own decision function, $f_{i}(\cdot)$. If there are $m$ different classifiers, there will be $m$ different decision functions. The goal of constructing a binary classifier, $\Phi(\cdot)$, is to approximate the unknown true target function $\breve{\Phi}(\cdot)$, so that $\Phi(\cdot)$ and $\breve{\Phi}(\cdot)$ are coincident as much as possible [17].

In order to improve the effectiveness, ensemble classifiers (a.k.a classifier committee) were proposed [1, 3, 5, 6, $7,8,9,15,16,17,18,19]$. An ensemble classifier is constructed by grouping a number of member classifiers. If the decisions of the member classifiers are combined properly, the ensemble is robust and effective. There are two kinds of ensemble classifiers: homogeneous and heterogeneous.

A homogeneous ensemble classifier contains $m$ binary classifiers in which all classifiers are constructed by the same learning algorithm. Bagging and boosting [19] are two common techniques $[1,15,16,18]$.

A heterogeneous ensemble classifier contains $m$ binary classifiers in which all classifiers are constructed by different learning algorithms (e.g., one SVM classifier and one $k \mathrm{NN}$ classifier are grouped together) [19]. The individual decisions of the classifiers in the ensemble are combined (e.g., through stacking [19]):

$$
\Theta(d)= \begin{cases}1 & \text { if } g\left(\Phi_{1}(d), \Phi_{2}(d), \ldots, \Phi_{m}(d)\right)>0, \\ -1 & \text { otherwise }\end{cases}
$$

where $\Theta(\cdot)$ is an ensemble classifier; $g(\cdot)$ is a combination function that combines the outputs of all $\Phi_{i}(\cdot)$. The effectiveness of the ensemble classifier, $\Theta(\cdot)$, depends on the effectiveness of $g(\cdot)$. In this paper, we concentrate on analyzing heterogeneous ensemble classifiers. Our problem is thus to examine how to formulate a good $g(\cdot)$.

Four widely used $g(\cdot)$ are: (1) Majority voting (MV) [8, 9]; (2) Weighted linear combination (WLC) [7]; (3) Dynamic classifiers selection (DCS) $[3,8,6,5]$; and (4) Adaptive classifiers combination (ACC) $[8,9]$. Except for MV, the other three functions assign different weights to the classifiers in the ensemble. The bigger the weight, the more ef- 


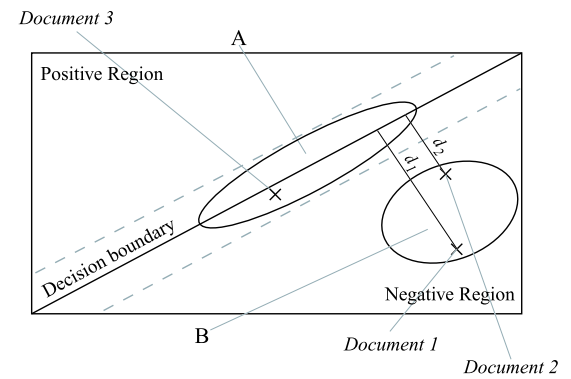

Figure 1. Illustration of local effectiveness and decision confidence.

fective is that classifier. In MV, all classifiers in the ensemble are equally weighted. It can end up with a wrong decision if the minority votes are significant. WLC assigns static weights to the classifiers based on their performance on a validation data. However, a generally well-performed classifier can perform poorly in some specific domains. For instance, the micro- $\mathrm{F}_{1}$ scores of SVM and Naive Bayes (NB) for the benchmark Reuters 21578 are respectively 0.860 and 0.788 . In this sense, SVM excels NB. Yet, for the categories Potato and Retail in Reuters21578, the $\mathrm{F}_{1}$ scores for NB are both 0.667 , but are both 0.0 for SVM. DCS and ACC weight the classifiers by partitioning the validation data (domain specific), they do not combine the classifiers' decisions, but select one of the classifiers from the ensemble and rely on it solely. We will show in the experiments that this will lead to inferior results.

In this paper, we propose a new combination function called Dynamic Classifiers Weighting (DCW). We consider three components when combining classifiers: (1) Global Effectiveness, which is the effectiveness of a classifier in an ensemble when it classifies a set of unseen documents; (2) Local effectiveness, which is the effectiveness of a classifier in an ensemble when it classifies the particular domain of the unseen document; and (3) Decision confidence, which is the confidence of a classifier in making a decision of the ensemble for a specific unseen document.

\section{Motivations}

Let $\Phi_{1}(\cdot), \Phi_{2}(\cdot), \ldots, \Phi_{m}(\cdot)$ be $m$ different binary classifiers and $f_{1}(\cdot), f_{2}(\cdot), \ldots, f_{m}(\cdot)$ be their corresponding decision functions. Conceptually, $\Phi_{i}(\cdot)$ divides the entire domain into two parts according to $f_{i}(\cdot)$. Figure 1 illustrates this idea. The dashed lines are the decision boundaries. If the unseen document, $d$, falls into the upper (lower) triangle, it would be labeled as positive (negative). Usually, if $d$ is further away from the decision boundary, the decision of $d$ by $\Phi_{i}(d)$ is more confident.

Every classifier has different effectiveness. For instance, Support Vectors Machine (SVM) is being regarded as more accurate (effective) than Naive Bayes (NB) [20]). Although it does not imply all of the decisions made by SVM must be superior than NB, it does imply that we should value the judgment of SVM higher than that of NB in general. In this paper, we term this kind of effectiveness as global effectiveness of a classifier, denoted by $\alpha_{i}$ (E.g. $\left.\alpha_{\mathrm{SVM}}>\alpha_{\mathrm{NB}}\right) . \alpha_{i}$ gives us good insight about how to weight the classifiers in an ensemble. Intuitively, if we construct an ensemble classifier by grouping $\Phi_{a}(\cdot)$ and $\Phi_{b}(\cdot)$ together, where $\alpha_{a}>\alpha_{b}$, then we should value $\Phi_{a}(\cdot)$ higher than $\Phi_{b}(\cdot)$.

Yet, a globally effective classifier may sometimes perform poorly on some specific dataset (domain). As an example, consider two classifiers, SVM and NB. According to the benchmark Reuters 21578 , the micro- $\mathrm{F}_{1}$ scores for SVM and NB are respectively 0.860 and 0.788 . Unfortunately, the $\mathrm{F}_{1}$ score for $\mathrm{SVM}$ when classifying Retail (Retail $\subset$ Reuters 21578 ) is 0.0 , but it is 0.667 for NB. As a result, an effective classifier may not always perform well in all domains (e.g., SVM performs poorly in Retail). This can be further illustrated in Figure 1. The two ovals, A and B, represent two different domains. Oval A covers over the decision boundary, whereas Oval B resides in the lower triangle. All of the documents within the domain of Oval A are aligned near the decision boundary. An unseen document that belongs to this domain may easily be classified wrongly. On the other hand, the documents within the domain of Oval B are well separated by the decision boundary. An unseen document that belongs to this domain will most likely be classified correctly. So, the effectiveness of the classifier also relies on the domain of the unseen data. We term this kind of effectiveness as local effectiveness of the classifier, denoted by $\beta_{i} . \beta_{i}$ helps us to adjust the weights of the classifiers in the ensemble. If the $\alpha_{i}$ of $\Phi_{i}$ is very high but it is not effective in classifying the domain of the unseen document, we should re-consider its effectiveness.

For every decision a classifier makes, one may ask how confident the classifier is about the decision? Consider the two unseen documents, document 1 and document 2 , in the same domain (Oval B) in Figure 1. While both document 1 and document 2 reside near the boarder of their domain, document 2 locates closer to the decision boundary (the dashed line) whereas document 1 locates far away from it. Since both document 1 and document 2 belong to the same domain, the local effectiveness of the classifier upon them are the same. Yet, the confidence in making a correct decision for document 1 should be higher than that of document 2 , as document 1 is further away from the decision boundary $\left(d_{1}>d_{2}\right)$. In this paper, we term it as decision confidence. It is estimated according to the distance between the unseen document and the decision boundary.

We summarize the needs for the above components as follows: if we ignore $\alpha_{i}$, over-fitting may result as we neglect the combined influence of all domains. If we ignore 
$\beta_{i}$, over-generalization may ensue as it relies on the domain where the unseen document appears. $\alpha_{i}$ and $\beta_{i}$ do not measure the classifier's decision confidence, $\gamma_{i}$ is proposed as it indicates how much confidence a classifier has when it classifies the unseen documents.

\section{Dynamic Classifiers Weighting (DCW)}

In the previous section, we have explained why the three weight components $\left(\alpha_{i}, \beta_{i}\right.$ and $\left.\gamma_{i}\right)$ are helpful in constructing an effective combination function, $g(\cdot)$. We now describe how they are estimated and how they are combined in an ensemble classifier.

$\alpha_{i}$ is the effectiveness of the classifier when we use it to classify a set of unseen documents. During the training phase, although we do not have a set of labeled unseen documents, we can estimate $\alpha_{i}$ from the training data, $\mathcal{D}$ : we estimate $\alpha_{i}$ by 10 -folded cross validation. While our experience suggested that estimating the effectiveness of a classifier based on cross validation would always yield an optimistic result than evaluating it from the unseen data, this would not be a problem in our situation, as we are not targeting for evaluating the real global effectiveness of the classifiers, but aiming at obtaining the relative global effectiveness. We normalize $\alpha_{i}$ such that $0<\alpha_{i}<1$ and $\sum_{i=1}^{m} \alpha_{i}=1$.

$\beta_{i}$ is the effectiveness of the classifier when we use it to classify the domain of the unseen document, $d$. For an unseen document, we would never know what the true domain of $d$ is. As above, we can only estimate its domain according to the training data, $\mathcal{D}$. Let $D$ be a subset of documents in the training data, i.e., $D \subseteq \mathcal{D}$. We can find the domain of the unseen document, $d$, by using $D$, to extract the documents in $D$ that are similar to $d$. Accordingly, the extraction of $D$ is based on a nearest neighbor strategy. We extract the top $n$ documents that are most similar to $d$ from $\mathcal{D}$. The value $n$ can be readily obtained through a validation dataset. The similarities among these $n$ documents are measured by the cosine coefficient [13]. Since $D$ is a subset of the training data $(D \subseteq \mathcal{D})$, we will know precisely the labels of those documents that appear in $D$. We estimate $\beta_{i}$ by evaluating $D$ using the $\mathrm{F}_{1}$ score. $\beta_{i}$ is normalized such that $0<\beta_{i}<1$ and $\sum_{i=1}^{m} \beta_{i}=1$.

$\gamma_{i}$ is a measure about how confident the classifier is when it makes a decision upon $d$. From Eq. (1), the classification decision of the classifier, $\Phi_{i}(\cdot)$, is based on the decision function, $f_{i}(\cdot)$. For most cases, if not all, the higher the magnitude of $f_{i}(\cdot)$, the more confident are their decisions. Consequently, we can compute $\gamma_{i}$ by using the decision function, $f_{i}(\cdot)$. Unfortunately, the range of $f_{i}(\cdot)$ varies among different algorithms. For example, $\Phi_{i}(\cdot)$ may have $f_{i}(\cdot)$ in the range of $[-1,1]$, whereas $\Phi_{j}(\cdot)$ may have another $f_{j}(\cdot)$ in the range of $(-\infty,+\infty)$. Since different decision functions have different ranges, a direct comparison among them is inappropriate. We solve the problem as follows: Let $D$ be the domain of the unseen document. $D$ is obtained by the technique described previously. We compute $\gamma_{i}$ as follows:

$$
\begin{aligned}
\gamma_{i} & =\left|\frac{f_{i}(d)}{\mu_{i}}\right|, \\
\mu_{i} & =\frac{1}{|D|} \sum_{d^{\prime} \in D} f_{i}\left(d^{\prime}\right),
\end{aligned}
$$

where $\mu_{i}$ is the average confidence of the decisions made by $f_{i}(\cdot)$ among the documents in $D$. Since $D \subseteq \mathcal{D}$, we can presume that $\mu_{i}$ is non-zero. When $\gamma_{i}>1, f_{i}(d)$, has more than average confidence to make a correct classification on $d$, where $d$ will be far away from the decision boundary (e.g., document 1 in Figure 1). When $\gamma_{i}<1$, the decision function, $f_{i}(d)$, has less than average confidence to make a correct classification on $d$, where $d$ will be closer to the decision boundary (e.g., document 2 in Figure 1). We normalize $\gamma_{i}$ such that $0<\gamma_{i}<1$ and $\sum_{i=1}^{m} \gamma_{i}=1$.

We now present how $\alpha_{i}, \beta_{i}$ and $\gamma_{i}$ are combined. Assume that there are $m$ classifiers in the ensemble. In the most simplest form, the combination function, $g(\cdot)$ is:

$$
g(\cdot)=\sum_{i}^{m} \text { decision }_{i},
$$

where decision $_{i}=\Phi_{i}(d) \in\{1,-1\}$ (Eq. (eq:c)). Here, all classifiers in the ensemble are equally weighted (i.e. MV). In DCW, since a confidence $\left(\gamma_{i}\right)$ is associated with each decision $_{i}$, therefore:

$$
g(\cdot)=\sum_{i}^{m} \operatorname{decision}_{i} \times \gamma_{i} .
$$

Yet, even for a confident decision, we need to review whether the classifier, which makes this decision, is effective in the ensemble. Consequently:

$$
g(\cdot)=\sum_{i}^{m} \operatorname{decision}_{i} \times \gamma_{i} \times \text { effectiveness }_{i} .
$$

Since there are two kinds of effectiveness for each of the classifier $\left(\alpha_{i}\right.$ and $\left.\beta_{i}\right)$, we have:

$$
g(\cdot)=\sum_{i}^{m}\left(\Phi_{i}(d) \times \alpha_{i} \times \beta_{i} \times \gamma_{i}\right),
$$

\section{Experimental Study}

The purpose of the experiments is twofold. (1) We want to examine how effective the Dynamic Classifiers Weighting $(\mathrm{DCW})$ is, when it is compared with the other kinds of heterogeneous ensemble classifiers. As such, we implemented four existing ensemble classifiers for comparison: 


\begin{tabular}{|c|c||c|c|c|c|c||c|c|c|c|c|}
\hline \multirow{2}{*}{ No. } & \multicolumn{1}{|c||}{ Combination } & \multicolumn{4}{|c||}{ Reuters21578 } & \multicolumn{4}{c|}{ Newsgroup20 } \\
\cline { 3 - 12 } & & MV & WLC & DCS & ACC & DCW & MV & WLC & DCS & ACC & DCW \\
\hline \hline 1 & $\mathrm{~S}+\mathrm{N}$ & - & 0.874 & 0.859 & 0.852 & $\mathbf{0 . 8 7 6}$ & - & 0.817 & 0.761 & 0.794 & $\mathbf{0 . 8 1 7}$ \\
\hline 2 & $\mathrm{~S}+\mathrm{R}$ & - & 0.883 & 0.862 & 0.874 & $\mathbf{0 . 8 8 5}$ & - & 0.800 & 0.762 & 0.793 & $\mathbf{0 . 8 0 0}$ \\
\hline 3 & $\mathrm{~S}+\mathrm{K}$ & - & 0.862 & 0.862 & 0.843 & $\mathbf{0 . 8 6 3}$ & - & 0.813 & 0.763 & 0.780 & $\mathbf{0 . 8 1 5}$ \\
\hline 4 & $\mathrm{R}+\mathrm{N}$ & - & 0.833 & 0.831 & 0.821 & $\mathbf{0 . 8 3 4}$ & - & 0.762 & 0.738 & 0.759 & $\mathbf{0 . 7 6 5}$ \\
\hline 5 & $\mathrm{~K}+\mathrm{N}$ & - & 0.824 & 0.827 & 0.820 & $\mathbf{0 . 8 2 9}$ & - & 0.780 & 0.746 & 0.769 & $\mathbf{0 . 7 8 0}$ \\
\hline 6 & $\mathrm{~K}+\mathrm{R}$ & - & 0.825 & $\mathbf{0 . 8 3 2}$ & 0.821 & $\mathbf{0 . 8 3 1}$ & - & 0.762 & 0.764 & 0.760 & $\mathbf{0 . 7 6 5}$ \\
\hline 7 & $\mathrm{~S}+\mathrm{K}+\mathrm{R}$ & 0.872 & 0.879 & 0.862 & 0.876 & $\mathbf{0 . 8 8 2}$ & 0.776 & 0.815 & 0.763 & 0.812 & $\mathbf{0 . 8 1 6}$ \\
\hline 8 & $\mathrm{~S}+\mathrm{K}+\mathrm{N}$ & 0.855 & 0.874 & 0.859 & 0.865 & $\mathbf{0 . 8 7 3}$ & 0.783 & 0.819 & 0.762 & 0.809 & $\mathbf{0 . 8 2 1}$ \\
\hline 9 & $\mathrm{~S}+\mathrm{R}+\mathrm{N}$ & 0.852 & 0.872 & 0.861 & 0.856 & $\mathbf{0 . 8 7 4}$ & 0.777 & $\mathbf{0 . 8 1 5}$ & 0.761 & 0.801 & $\mathbf{0 . 8 1 5}$ \\
\hline 10 & $\mathrm{~K}+\mathrm{R}+\mathrm{N}$ & 0.857 & 0.825 & 0.837 & 0.823 & $\mathbf{0 . 8 3 0}$ & 0.775 & 0.782 & 0.750 & 0.775 & $\mathbf{0 . 7 8 4}$ \\
\hline 11 & $\mathrm{~S}+\mathrm{K}+\mathrm{R}+\mathrm{N}$ & - & 0.851 & 0.861 & 0.859 & $\mathbf{0 . 8 5 3}$ & - & 0.720 & 0.762 & 0.761 & $\mathbf{0 . 7 6 3}$ \\
\hline
\end{tabular}

Table 1. The results of the micro- $\mathrm{F}_{1}$ for different ensemble classifiers.

Majority voting (MV) [8, 9], Weighted linear combination (WLC) [7], Dynamic classifiers selection (DCS) [3, 8, 6, 5], and Adaptive classifiers combination (ACC) $[8,9]$. We report the results in Section 4.1. (2) We want to understand how significant the results are whenever one of the ensemble classifiers outperforms the others. As such, we performed a pairwise significant test in Section 4.2.

In the experiments, two benchmarks are used: Reuters21578 and Newsgroup20. For Reuters21578, we separate the dataset into training data and testing data using the ModApte split [2]. For Newsgroup20, for each of the categories, we randomly select $80 \%$ of the postings as training data, and the remaining as testing data.

For the data preprocessing, punctuation, numbers, web page addresses, and email addresses are removed. All features are stemmed and converted to lower cases, and are weighted using the standard $t f \cdot$ idf schema [14]. Features that appear in only one document are ignored. All features are ranked based on the NGL Coefficient[12], and the top $X$ features are selected. This $X$ is tuned for different classifiers and for different benchmarks.

For creating the ensemble classifiers, different combinations of four kinds of classifiers are used: (1) Support Vectors Machine (SVM); (2) $k$-Nearest Neighbor $(k N N)$; (3) Rocchio (ROC); (4) Naive Bayes (NB). Their default settings are as follows: For SVM, we use linear kernel with $C=1.0$. No feature selection is required [4]. For $k \mathrm{NN}$, we set $k=50$ and select 2,750 and 4,900 features for Reuters 21578 and Newsgroup20. For ROC, we implement the version in [11] and selects 2,750 and 7,500 features for Reuters 21578 and Newsgroup20. For NB, we implement the multinomial version [10] and selects 2,750 and 9,500 features for Reuters 21578 and Newsgroup20.

\subsection{Effectiveness Analysis}

Table 1 shows the results of the micro- $\mathrm{F}_{1}$ score for all ensemble classifiers (MV, WLC, DCS, ACC and DCW) when they are created using different combinations of the binary classifiers for both benchmarks. The left most column denotes which of the binary classifiers are used for creating the corresponding ensemble classifier. We use S, K, R and $\mathrm{N}$ to denote SVM, $k N N$, Rocchio and Naive Bayes, respectively. For example, $\mathrm{S}+\mathrm{K}+\mathrm{R}$ represents an ensemble classifier which is comprised of SVM, $k \mathrm{NN}$ and Rocchio. Note that MV cannot be created if the number of binary classifiers in the ensemble is an even number, hence the "-" entries in Table 1.

At the first glance, the results are promising. DCW, the proposed approach, dominates over all other approaches when they are being created using the same set of binary classifiers. Similar results are obtained when we use the macro- $F_{1}$ score. The only case where DCW performs inferior is case 6 when DCW is created by $k \mathrm{NN}$ and Rocchio $(\mathrm{K}+\mathrm{R})$, meanwhile it is evaluated using Reuters 21578 . Its micro- $\mathrm{F}_{1}$ is 0.831 , which is 0.001 lower than DCS (Dynamic Classifiers Weighting). Nevertheless, such a difference can be negligibled.

Concerning DCW, the best combination of binary classifiers in the ensemble is SVM and Rocchio (case 2) for Reuters 21578. The micro- $\mathrm{F}_{1}$ score is 0.885 . It is also the best results obtained among all of the ensemble classifiers that we have evaluated. For Newsgroup20 the best result is obtained by comprising SVM, $k \mathrm{NN}$ and Rocchio together (case 8 ). The micro- $F_{1}$ score is 0.821 . It is also the best result obtained among all approaches.

For MV, its philosophy is to take the majority agreement among the binary classifiers in the ensemble. Hence, the number of binary classifiers must be an odd number. So we can only create MV using three different binary classifiers. Interestingly, all combinations perform similarly.

Concerning WLC, the best combination for Reuters 21578 (case 2), its micro- $F_{1}$ score is 0.883 , which is higher than all ensemble classifiers (except DCW). For Newsgroup20, similar observations are made, where its best combination is case 8 . Although the idea of WLC is very simple - assigns static weights to the classifiers in the ensemble according to their global effectiveness and 
combines them linearly - it performs surprisingly well. Another interesting finding is that when SVM is included in the ensemble, the effectiveness of WLC would be increased dramatically. This suggests that the choice of the classifiers in WLC is particularly important.

Concerning DCS, its best micro-F $F_{1}$ score for Reuters 21578 (case 2) is 0.862 only. It is far lag behind all the other approaches. For Newsgroup20, none of the $\mathrm{F}_{1}$ score is higher than 0.77 . We believe that the reasons of why DCS performs poorly are because: (1) It does not combine the classifiers' decisions. Rather, it selects one of the classifier in the ensemble and relies on it completely. (2) It neither pays attention to the global effectiveness of the classifiers nor the decision confidence.

ACC performs slightly better than DCS. This may be because the decision strategy for ACC is more sophisticated that DCS. The best ensembles for Reuters 21578 and Newsgroup 20 are both case 7 . However, these results are all inferior than both WLC and our DCW.

\subsection{Significant Test}

In this section, we conduct a pairwise comparison among them using the significant test [20]. Given two classifiers, $\Phi_{A}(\cdot)$ and $\Phi_{B}(\cdot)$, the significant test determines whether $\Phi_{A}(\cdot)$ performs better than $\Phi_{B}(\cdot)$ based on the errors that $\Phi_{A}(\cdot)$ and $\Phi_{B}(\cdot)$ made. Let $N$ be the total number of the unseen documents, and $a_{i}=\{0,1\}\left(b_{i}=\{0,1\}\right)$ indicate whether $\Phi_{A}(\cdot)\left(\Phi_{B}(\cdot)\right)$ makes a correct classification upon the $i^{t h}$ unseen document. $a_{i}=0$ means $\Phi_{A}(\cdot)$ makes an incorrect classification whereas $a_{i}=1$ means $\Phi_{A}(\cdot)$ makes a correct one. Similar definition is also applied to $b_{i}$. Let $d_{a}$ be the number of times that $\Phi_{A}(\cdot)$ performs better than $\Phi_{B}(\cdot)$, and $d_{b}$ be the number of times that $\Phi_{B}(\cdot)$ performs better than $\Phi_{A}(\cdot)$. In this test, the null hypothesis is that both classifiers perform the same $\left(H_{0}: d_{a}=d_{b}\right)$. The alternative is that $\Phi_{A}(\cdot)$ and $\Phi_{B}(\cdot)$ performs differently $\left(H_{1}: d_{a} \neq d_{b}\right)$.

Table 2 shows the results of comparing the performance of DCW with the other ensemble classifiers. $A \gg B$ means $A$ performs significantly better than $B(P$-Value $\leq 0.01)$. $A>B$ means $A$ performs slightly better than $B . A \sim B$ means no evidence indicates $A$ and $B$ has any differences in terms of the errors they made. A summary is given below:

$$
\begin{aligned}
& \text { Reuters21578: }\{\mathrm{DCW}, \mathrm{WLC}\}>\{\mathrm{MV}, \mathrm{ACC}\} \gg \mathrm{DCS} \\
& \text { Newsgroup20: } \mathrm{DCW}>\mathrm{WLC}>\mathrm{ACC} \gg \mathrm{MV} \gg \mathrm{DCS}
\end{aligned}
$$

\section{Conclusions}

In order to formulate an effective combination function for heterogeneous ensemble classifier, three weight components are necessary: Global Effectiveness, Local Effectiveness, and Decision Confidence. We compare DCW with

\begin{tabular}{|c|c|c|c|}
\hline A & B & Reuters21578 & Newsgroup20 \\
\hline \hline MV & WLC & $<$ & $\ll$ \\
\hline MV & DCS & $\gg$ & $\gg$ \\
\hline MV & ACC & $\sim$ & $\ll$ \\
\hline MV & DCW & $\ll$ & $\ll$ \\
\hline \hline WLC & DCS & $\gg$ & $\gg$ \\
\hline WLC & ACC & $>$ & $>$ \\
\hline WLC & DCW & $\sim$ & $<$ \\
\hline \hline DCS & ACC & $\ll$ & $\ll$ \\
\hline DCS & DCW & $\ll$ & $\ll$ \\
\hline \hline ACC & DCW & $<$ & $\ll$ \\
\hline
\end{tabular}

Table 2. Results of the significant test.

four other kinds of heterogeneous ensemble classifiers using two benchmarks. The results indicated that DCW can effectively balance the contributions of the three components and outperforms the existing approaches.

\section{References}

[1] W. W. Cohen and Y. Singer. Context-sensitive learning methods for text categorization. ACM Transactions on Information Systems (TOIS), 17(2):141-173, 1999.

[2] F. Debole and F. Sebastiani. An analysis of the relative hardness of Reuters-21578 subsets. Journal of the American Society for Information Science and Technology, 56(6):584-596, 2004.

[3] G. Giacinto and F. Roli. Adaptive selection of image classifiers. In Proceedings of the 9th International Conference on Image Analysis and Processing (ICIAP'97), pages 38-45, Florence, Italy, 1997.

[4] T. Joachims. Text categorization with support vector machines: Learning with many relevant features. In Proceedings of 10th European Conference on Machine Learning (ECML'98), pages 137-142, Chemnitz, Germany, 1998.

[5] K. B. Kevin Woods, W. Philip Kegelmeyer. Combination of multiple classifiers using local accuracy estimates. IEEE Transactions on Pattern Analysis and Machine Intelligence (TPAMI), 19(4):405-410, 1997.

[6] W. Lam and K.-Y. Lai. A meta-learning approach for text categorization. In Proceedings of the 24th Annual International ACM SIGIR Conference on Research and Development in Information Retrieval (SIGIR'01), pages
303-309, New Orleans, Louisiana, USA, 2001 .

[7] L. S. Larkey and W. B. Croft. Combining classifiers in text categorization. In Proceedings of the 19th Annual International ACM SIGIR Conference on Research and Development in Information Retrieval (SIGIR' 96 ), pages 289-297, Zurich, Switzerland, 1996.

[8] Y. H. Li and A. K. Jain. Classification of text documents. The Computer Journal, 41(8):537-546, 1998.

[9] R. Liere and P. Tadepalli. Active learning with committees for text categorization. In Proceedings of 14th National Conference on Artificial Intelligence (AAAI'97), pages 591-596, Providence, Rhode Island, 1997.

[10] A. McCallum and K. Nigam. A Comparison of Event Models for Naive Bayes Text Classification. In The 15th National Conference on Artificial Intelligence (AAAI' 98 ) Workshop on Learning for Text Categorization, 1998.

[11] A. Moschitti. A study on optimal parameter tuning for rocchio text classifier. In Proceedings of the 25th European Conference on Information Retrieval Research (ECIR'03), pages 420-435, Pisa, Italy, 2003.

[12] H. T. Ng, W. B. Goh, and K. L. Low. Feature selection, perception learning, and a usability case study for text categorization. In Proceedings of the 20th Annual International ACM SIGIR Conference on Research and Development in Information Retrieval (SIGIR'97), pages 67-73, Philadelphia, PA, USA, 1997.

[13] E. Rasmussen. Clustering algorithm. In W. B. Freakes and R. Baeza-Yates, editors, Information Retrieval Data Structures \& Algorithms, pages 419-442. Prentice Hall PTR, 1992

[14] G. Salton and C. Buckley. Term-weighting approaches in automatic text retrieval. Information Processing and Management (IPM), 24(5):513-523, 1988

[15] R. E. Schapire and Y. Singer. BoosTexter: a boosting-based system for text categorization. Machine Learning, 39(2-3): 135-168, 2000 .

[16] R. E. Schapire, Y. Singer, and A. Singhal. Boosting and Rocchio applied to text filtering. In Proceedings of the 2Ist Annual International ACM SIGIR Conference on Research and Development in Information Retrieval (SIGIR'98), pages 215-223, Melbourne, Australia, 1998.

[17] F. Seabastiani. Machine learning in automated text categorization. ACM Computing Surveys, 34(1):1-47, 2002.

[18] S. M. Weiss, C. Apte, F. J. Damerau, D. E. Johnson, F. J. Oles, T. Goetz, and T. Hampp. Maximizing text-mining performance. IEEE Intelligent Systems, 14(4):63-69, 1999.

[19] I. H. Witten and E. Frank. Data Mining: Practical Machine Learning Tools and Techniques. Morgan Kaufmann, second edition, 2005.

[20] Y. Yang and X. Liu. A re-examination of text categorization methods. In Proceedings of the 22nd Annual International ACM SIGIR Conference on Research and Development in Information Retrieval (SIGIR'99), pages 